\title{
Evolución del daño mecánico del concreto SFRC sometido a flexión mediante el análisis de la velocidad del pulso ultrasónico
}

\section{Evolution of the mechanical damage of concrete SFRC under flexion load through the analysis of the speed of the ultrasonic pulse}

DOI: http://doi.org/10.17981/ingecuc.16.1.2020.15

Artículo de Investigación Científica. Fecha de Recepción: 05/05/2019. Fecha de Aceptación: 05/02/2020

\author{
Miguel Ángel Ospina García (1) \\ Universidad Militar Nueva Granada. Bogotá, D.C. (Colombia) \\ miguel.ospina@unimilitar.edu.co \\ Juan Manuel Lizarazo Marriaga \\ Universidad Nacional de Colombia. Bogotá, D.C. (Colombia) \\ jmlizarazom@unal.edu.co \\ Andrés Salas Montoya (D) \\ Universidad Nacional de Colombia. Bogotá, D.C. (Colombia) \\ asalasm@unal.edu.co
}

Para citar este artículo:

M. Ospina García, J. Lizarazo Marriaga y A. Salas Montoya, "Evolución del daño mecánico del concreto SFRC sometido a flexión mediante el análisis de la velocidad del pulso ultrasónico", INGE CUC, vol. 16, no. 1, pp. 205-216, 2020. DOI: http://doi.org/10.17981/ingecuc.16.1.2020.15

\section{Resumen}

Introducción- En el presente artículo se realiza un análisis de la evolución del daño mecánico del Concreto Reforzado con Fibras de Acero (SFRC) y la Velocidad de Pulso Ultrasónico (UPV) propagada; con la finalidad de correlacionar el UPV y el daño mecánico en elementos de concreto con diferentes dosificaciones de fibras de acero. Para lo cual se fabricaron 45 probetas en concreto, con dosificaciones de $0 \mathrm{~kg} / \mathrm{m}^{3} ; 25 \mathrm{~kg} / \mathrm{m}^{3}$ y $70 \mathrm{~kg} / \mathrm{m}^{3}$, y resistencias promedio a la compresión de $57 \mathrm{MPa}, 53 \mathrm{MPa}$ y $44 \mathrm{MPa}$ y a la flexión de 5,49 $\mathrm{MPa}, 7,20 \mathrm{MPa}$ y $9,60 \mathrm{MPa}$, esclareciendo que en los elementos probados se midieron carga, deflexión y velocidad de pulso ultrasónico en simultaneo. Obteniendo parámetros que permitieron generar ecuaciones predictivas del daño en una escala de 0 a 1 , teniendo en cuenta que un daño mecánico es avanzado cuando un elemento tiene una alta fisuración y por lo tanto se disminuirá su capacidad de resistencia mecánica (cuando el valor es cercano o igual a 1).

Objetivo- Estimar la evolución del daño mecánico del concreto tipo SFRC, mediante la medición de la velocidad de pulso ultrasónico, correlacionando con la resistencia a la flexión, obteniendo ecuaciones de predicción del daño y gráficas de comportamiento mecánico.

Metodología- Para la realización del proyecto se aplica un modelo experimental, iniciando con una búsqueda primaria de información sobre concretos fibro-reforzado y métodos de inspección indirecta. Se definió las dosificaciones y mezclas de concreto que se van a realizar y se fabrica los elementos de prueba, realizando pruebas experimentales y analizando sus resultados.

Resultados-Se obtiene ecuaciones predictivas y gráficas de comportamiento mecánico, las cuales permitirán que al inspeccionar un elemento de concreto simple y concreto SFRC, se obtenga en primera instancia, una idea del daño mecánico del concreto, lo que permitiría establecer el estado físico en el que se encuentra un elemento fabricado en este material.

Conclusiones- A medida que se presente daño mecánico en un elemento de concreto, estos aumentarán su fisuración y por lo tanto su densidad disminuirá; esta característica se puede medir con la velocidad de pulso propagada; consiguiente la predicción de la integralidad del estado físico en el que se encuentra un elemento será predecible, permitiendo conocer si una estructura en concreto se encuentra en riesgo de colapso o cercano al mismo. Lo anterior cobra mayor importancia en estructuras sometidas a flexión (propiedad en la cual el concreto ofrece baja resistencia, con respecto a la resistencia a la compresión), tal como un puente, una vía vehicular o vigas de una edificación. Con las ecuaciones expuestas, se demuestra que en un daño mecánico inferior a 0,5 , la estructura se fisura, pero no colapsará, en cambio si el daño es superior a 0,5 la estructura se encuentra tan fisurada que no podrá soportar la carga máxima de diseño y por lo cual puede colapsar. De la misma manera, en el proyecto se demuestra que las fibras de acero aumentan la resistencia a la flexión y tenacidad del concreto en por lo menos un $30 \%$, lo que implica su utilidad para la construcción de elementos descritos anteriormente.

Palabras clave- Velocidad de pulso ultrasónico; daño mecánico; evolución del daño; Concreto reforzado con fibras de acero SFRC, métodos indirectos

\section{Abstract}

Introduction- In this article, an analysis of the evolution of the mechanical damage of Steel Fiber Reinforced Concrete (SFRC) and propagated Ultrasonic Pulse Velocity (UPV) is performed; in order to correlate the UPV and mechanical damage in concrete elements with different dosages of steel fibers. For which 45 concrete samples were manufactured, with dosages of $0 \mathrm{~kg} / \mathrm{m}^{3} ; 25 \mathrm{~kg} / \mathrm{m}^{3}$ and $70 \mathrm{~kg} / \mathrm{m}^{3}$, and average compressive strengths of $57 \mathrm{MPa}, 53 \mathrm{MPa}$ and $44 \mathrm{MPa}$ and flexural strength of 5.49 $\mathrm{MPa}, 7.20 \mathrm{MPa}$ and $9.60 \mathrm{MPa}$, is to clarifying that in the tested elements load, deflection and ultrasonic pulse velocity were measured in simultaneously. Obtaining parameters that allowed to generate predictive equations of the damage, on a scale of 0 to 1 , taking into account that a mechanical damage is advanced when an element has a high cracking and therefore its mechanical resistance capacity will be reduced (when the value is close or equal to 1 )

Objective- Estimate the evolution of the mechanical damage of the SFRC-type concrete, by measuring the ultrasonic pulse velocity, correlating with the flexural strength, obtaining damage prediction equations and graphs of mechanical behavior.

Methodology-An experimental model is applied to the project, starting with a primary search for information on fiber-reinforced concrete and indirect inspection methods. The dosages and concrete mixtures to be made were defined and the test elements were manufactured, carrying out experimental tests and analyzing their results.

Results- Predictive and graphical equations of mechanical behavior are obtained, which will allow an insight of the mechanical damage of the concrete to be inspected in the first instance, which would allow establishing the physical state in the that an element made of this material is found.

Conclusions-As mechanical damage occurs in a concrete element, these will increase its cracking and therefore its density will decrease; This feature can be measured with the pulse rate propagated; consequently the prediction of the integrality of the physical state in which an element is found will be predictable, allowing to know if a particular structure is at risk of collapse or close to it. The foregoing becomes more important in structures subjected to flexion (property in which the concrete offers low resistance, with respect to compressive strength), such as a bridge, a vehicular track or beams of a building. With the equations set forth, it is shown that in a mechanical damage of less than 0.5 , the structure cracks, but will not collapse, but if the damage is greater than 0.5 the structure is so fissured that it will not be able to withstand the load maximum design and therefore can collapse. In the same way, the project shows that steel fibers increase the flexural strength and toughness of the concrete by at least $30 \%$, which implies its usefulness for the construction of elements described above.

Keywords- Ultrasonic pulse velocity; mechanical damage; evolution of damage; reinforced concrete with SFRC steel fibers, indirect methods 


\section{INTRODUCCIÓN}

El concreto es uno de los materiales más importantes en la industria de la construcción [4], esto se debe a sus bondades constructivas de las cuales, una de las más importantes es su capacidad de combinarse con diferentes materiales de mejor comportamiento mecánico, tales como el acero o el polipropileno [5], mejorando sus características mecánicas; lo que comúnmente se conoce como concreto reforzado.

Al reforzar el concreto mediante la adición de fibras de acero, se mejora la resistencia a tensión y flexión del mismo, la cual llega a ser 4 veces la resistencia normal de éste [6], contrariamente, al aumentar el contenido de fibras de acero disminuye la resistencia a la compresión [7], por lo tanto se debe tener en cuenta esta consecuencia al momento de su uso.

De la misma manera, es necesario asegurar la calidad de los elementos fabricados con estos tipos de materiales; cuyos métodos principalmente se basan en las mediciones de las propiedades mecánicas; unas técnicas son de forma destructiva (se obtienen muestras o probetas testigos y estas se cargan hasta el colapso de las mismas), otras no destructivas, estás últimas con la ventaja que se realizan directamente en elemento a consideración sin dañarlo [8], sin embargo, su precisión depende de la cantidad de correlaciones entre la medición y las características del material a medir, por lo cual su medición es considerada como una aproximación más no una realidad, de todas maneras, estas mediciones son cercanas a los parámetros reales.

De estas técnicas la que ha tenido un mejor desarrollo son las de aplicación de ondas mecánicas, midiendo el tiempo que tardan de ir de un punto a otro; obteniendo la velocidad de propagación; dichas ondas se generan por ruido o por un golpe [9], esta técnica permite conocer la densidad del elemento, cantidad de vacíos, características físicas y humedad de un sólido, por lo cual si se emplea en el concreto permitirá conocer estas características físicas del mismo [10].

Con la medición de la velocidad de pulso ultrasónico se mide principalmente para saber a qué espesores, profundidades y densidades tiene un concreto en evaluación, sin embargo su uso para caracterizar mecánicamente un elemento en concreto no es común, debido a la alta variabilidad que presenta las mediciones mecánicas de un elemento fabricado en este material [11], sin embargo estas predicciones no son muy exactas y depende demasiado de las características de los áridos de dichos concretos; por lo cual las predicciones pueden servir si los elementos de fabricación del concreto son similares.

Igualmente, el analizar un elemento y poder intuir en qué momento se presentará su colapso o presentará debilitamiento mecánico (más conocido como daño mecánico), es conveniente; es sabido que un elemento presenta daño cuando se empieza a fisurar [3], característica que es más predecible en concreto reforzado que simple [10], lo cual provoca un impacto en su densidad, esto a su vez disminuiría la velocidad de propagación de una onda, de esta misma manera, las propiedades mecánicas del concreto se disminuirán, haciendo posible la correlación entre dichos parámetros, si estos se miden en el mismo instante de tiempo en el que se empieza a dañar un elemento.

Basado en lo anterior, se realiza un proyecto de investigación con la finalidad de medir estos parámetros en simultaneo; para lo cual se empleó una prensa de ensayos universal que permite medir carga y deflexión en elementos de concreto tipo viga sometidos a una carga en tercios medios de la luz; y un equipo de medición de la velocidad de pulso ultrasónico. Para garantizar que las mediciones fueran simultaneas, en los ensayos se controló la velocidad mediante la deflexión de los elementos en intervalos de tiempo, y en dichos instantes se midió la velocidad de onda propagada, para lo cual se empleó el software Data manager pc (de la maquina Controls MC8 de la Universidad Miltiar Nueva Granada) y el software de la maquina pundit lab de la empresa PROCEQ (equipo de la Universidad Nacional de Colombia).

Con la finalidad de obtener más parámetros se reforzó el concreto con fibras de acero a dosificaciones $0 \mathrm{~kg} / \mathrm{m} 3 ; 25 \mathrm{~kg} / \mathrm{m}^{3}$ y $70 \mathrm{~kg} / \mathrm{m}^{3}$; estas dosificaciones se escogen a partir de las recomendaciones de los fabricantes de fibras y normativas; y dos parámetros extremos para conocer su variabilidad; como resultado se obtuvo las ventajas de las fibras de acero en cuanto a la mejora en las propiedades mecánicas del concreto; esto se puede observar en las gráficas de comportamiento mecánico expuestas en este artículo; y se desarrollaron ecuaciones de predicción, que permitirán conocer el grado de daño mecánico de un elemento fabricado con este tipo de concreto; dichas ecuaciones permiten establecer un parámetro en una escala entre 0 a 1 ; donde 1 es un daño mecánico avanzado en los elementos. 
Para la realización de este artículo se desarrolló una metodología cuantitativa.

\section{Propiedades Físico-Mecánicas del concreto SFRC}

El concreto reforzado con fibras de acero permiten obtener las características mecánicas mejores con respecto a los otros y por lo tanto controlar su calidad, así como su uso. Autores como Song y hwang establecen que el concreto reforzado con fibras de acero llegan a duplicar la resistencia a la flexión [12], y el elemento llega a tener un comportamiento dúctil [13]; en este caso el agrietamiento es más visible y por lo tanto es más fácil de observar el daño de un elemento; a continuación se hará una descripción de las propiedades.

\section{A. Daño mecánico}

Para conocer la evolución del daño en un elemento, es necesario saber el comienzo y el final del proceso del mismo; por lo tanto, se establece que un material está libre de daño sí éste no posee fisuras visibles en un microscopio [3], cuando un elemento presenta un daño avanzado se pueden visualizar las fisuras a simple vista sin necesidad de un instrumento. Esto a su vez dependerá del tipo de material y su dureza, por ejemplo, las fisuras son visibles cuando se encuentran entre 0,1 mm - $1 \mathrm{~mm}$ en el acero, $10 \mathrm{~mm}$ en la madera y $100 \mathrm{~mm}$ en el concreto. Si se presentan estas fisuras en cualquiera de los elementos, significa que el daño es avanzado, por lo tanto, la debilidad mecánica del elemento es inminente.

Igualmente, el hecho que se presente daño en un elemento se puede atribuir a varios factores tales como cuando el material es sometido a esfuerzos mecánicos que superan su rango elástico, o cuando el material está sometido a temperaturas mayores de su rango de dilatación, o tienen un desgaste por su oxidación. Por lo tanto, las variables que originan el daño de un elemento pueden llegar a ser muy complejas; sin embargo, este se presenta principalmente por efectos de esfuerzos mecánicos a los que se someten los elementos.

\section{B. Análisis sobre el daño mecánico}

Según Lemaitre [3] daño es cuando un elemento empieza a fisurarse, dándose descohesión interatómica. Si se analiza los esfuerzos axiales en un elemento, se define que el daño se calcula con (1):

$$
D_{c}=1-{ }^{\sigma_{u}} \tilde{\sigma} u
$$

Donde Dc = Daño; $\sigma u$ = Esfuerzo uniaxial en la fractura del material; $\widetilde{\sigma u}=$ Esfuerzo último en la fractura del elemento. Si al aplicarse está ecuación el daño de un elemento se encuentra entre $0,5-0,9$; este se encuentra en un daño avanzado.

Basado en las variaciones de la velocidad propagada del concreto, se planteo (2) [3]; donde se define que $\widetilde{V}_{L}=$ Velocidad de onda en la fractura del elemento y $V_{L}=$ Velocidad de propagación de onda en el material sin daño.

$$
D_{c}=1-\tilde{V}_{L}^{2} / V_{L}^{2}
$$

Igual que (1); si los valores calculados se encuentran entre 0.5 - 1; el elemento tiene un daño avanzado.

\section{MetodoloGía}

\section{A. Dosificación de las fibras de acero y materiales participantes}

Para la realización del proyecto, se fabricaron 15 probetas de concreto simple y concreto reforzado con fibras de acero en vigas de sección cuadrada de $0,15 \mathrm{~m}$ y de 0,6 m de largo; 15 cilindros de concreto de $0,15 \mathrm{~m}$ de diámetro y una altura de $0,3 \mathrm{~m}$ como testigos de la resistencia a la compresión del concreto y 15 cilindros de $0,15 \mathrm{~m}$ de diámetro y una altura de $0,3 \mathrm{~m}$ 
para determinar la resistencia a la tensión. Estas cantidades se basan en tener 5 muestras por parámetro mecánico y mezcla de concreto; con la finalidad de obtener un promedio confiable en los resultados, en total se realizaron 45 probetas de ensayo. Todas se fabricaron el mismo día, con la misma relación agua material cementante y misma cantidad de materiales participantes.

En cuanto a las dosificaciones a realizar, y cómo variable para el proyecto, se decidió realizar tres tipos de mezclas reforzadas con fibras de acero (debido a que estas mezclas son más predecibles en su comportamiento y aumenta la ductilidad del concreto) las cuales se resume de la siguiente manera: una mezcla sin contenido de fibras, una con $25 \mathrm{~kg}$ por $\mathrm{m}^{3}$ de fibras y una con un contenido de $70 \mathrm{~kg}$ por $\mathrm{m}^{3}$.

Esta elección de dosificaciones, se basa en el estudio del estado del arte del proyecto, principalmente por recomendaciones de diferentes fabricantes de fibras tales como SIKA, TOXEMENT ó ALMASA, quienes sugieren emplear una cantidad de $20 \mathrm{~kg}$ a $45 \mathrm{~kg}$ de fibras por cada $\mathrm{m}^{3}$ de concreto; donde la cantidad dependerá del uso al elemento, por ejemplo, si es para pisos industriales con pocas juntas, se escoge el valor más alto; en cuanto al valor más bajo son para elementos de poca longitud y muy esbeltos.

A su vez la Norma Sismo Resistente Colombiana 10 en su título F numeral F.4.7.5.5. [20] recomienda emplear mínimo $14,8 \mathrm{~kg}$ por cada $\mathrm{m}^{3}$ de concreto para losas de concreto sin compromiso estructural. sin embargo, no se especifica un contenido máximo de estas fibras, ni contempla otro uso, tales como vigas o columnas, de lo anterior, un valor cercano al promedio de ambos criterios es de $25 \mathrm{~kg} / \mathrm{m}^{3}$. Este valor se recomienda para el uso de fibras de acero con la finalidad de mejorar la ductilidad del concreto.

Para la elección del límite superior de las mezclas, se escoge primero por recomendaciones de Ding y Wolfgang [14], quienes establecen un límite de $60 \mathrm{~kg}$ por cada $\mathrm{m}^{3}$ de concreto como un valor alto, proporcionando un aumento hasta un 50\% en la resistencia a la flexión. Como se deseaba conocer más incidencias, se decide llevarlo aún valor más alto y se define un límite de $70 \mathrm{~kg} / \mathrm{m}^{3}$, esperando que incrementará aún más la resistencia a la flexión del concreto.

Para el diseño de la mezcla de concreto, se empleó los parámetros y criterios establecidos por el código del American Concrete Institute, ACI 211.1-91 [2]. Para la cual se realizó la caracterización de los materiales empleados, resumidos en la Tabla 1, y en cuanto a las características físicas de las fibras empleadas se resumen en la Tabla 2.

La resistencia de diseño escogida para el proyecto es de $28 \mathrm{MPa}$ con un módulo de rotura equivalente de 4,23 $\mathrm{MPa}$, se elige esta resistencia para tener un concreto con una buena cantidad de cemento. En cuanto al asentamiento se elige que la mezcla sea plástica, para permitir un mejor moldeo y mezcla con las fibras; en el diseño se contempla el uso de superplastificante, con la finalidad de no usar una alta relación a/mc y se moldee de forma adecuada las probetas a fabricar, las cuales son esbeltas, tal como se muestra en la Fig. 1.

Tabla 1. Criterios escogidos para el diseño de la mezcla de concreto.

\begin{tabular}{|l|l|}
\hline \multicolumn{1}{|c|}{ Criterio } & \multicolumn{1}{c|}{ Dato } \\
\hline Resistencia a la Compresión de diseño & $28 \mathrm{MPa}$ \\
\hline Módulo de Rotura y Tensión & $4,23 \mathrm{MPa}$ \\
\hline Asentamiento & 8 in o $200 \mathrm{~mm}$ aprox. \\
\hline Densidad del cemento & $2,91 \mathrm{~g} / \mathrm{cm}^{3}$ \\
\hline Tamaño Máximo del Agregado & $11 / 2 \mathrm{in} \mathrm{o} 38,1 \mathrm{~mm}$ \\
\hline Tamaño Máximo Nominal del agregado & 1 in o $25,4 \mathrm{~mm}$ \\
\hline Masa unitaria Agregado Grueso & $1589 \mathrm{~kg} / \mathrm{m}^{3}$ \\
\hline Masa unitaria Agregado Fino & $1574 \mathrm{~kg} / \mathrm{m}^{3}$ \\
\hline Densidad Agregado Grueso & $2540 \mathrm{~kg} / \mathrm{m}^{3}$ \\
\hline Densidad Agregado Fino & $2510 \mathrm{~kg} / \mathrm{m}^{3}$ \\
\hline Módulo de finura & 3,1 \\
\hline
\end{tabular}

Fuente: Autores. 
Tabla 2. Datos técnicos de las fibras Sikafiber FE-530/35 GH.

\begin{tabular}{|l|l|}
\hline \multicolumn{1}{|c|}{ Descripción } & \multicolumn{1}{c|}{ Dato técnico } \\
\hline Longitud & $35 \mathrm{~mm}$ \\
\hline Diámetro de la fibra & $0,53 \mathrm{~mm}-0,54 \mathrm{~mm}$ \\
\hline Relación largo/diámetro (L/d) & 65 \\
\hline Resistencia a tracción & $1200 \mathrm{MPa}$ \\
\hline
\end{tabular}

Fuente: Basado en hoja técnica Sikafiber FE-530/35 GH.

Para la elección de las dosificaciones se definió como variable de las mezclas la cantidad de fibra, basado en el estudio del estado del arte, que entre mayor sea la cantidad de fibras de acero, mayor será la resistencia a la flexión y tensión del concreto [12]. Al observarse las características de los materiales participantes, es notable que no tienen características especiales, es decir el cemento es de uso general con adiciones (cemento comercial vendido en Colombia, donde los cementos contienen adiciones puzolanicas), por lo tanto su densidad es normal; el agregado empleado es de origen natural de roca sedimentaria (localmente conocido como canto rodado y arena de rio) obteniendo densidades típicas de estos materiales; y en cuanto a las fibras de acero, son las que se ofrecen comercialmente en Colombia, con una resistencia a la tensión de $1200 \mathrm{MPa}$, certificada por el fabricante, la relación 1/d son las recomendadas para su empleo en losas o vías o elementos esbeltos. No se recomienda otro uso, debido a que la normatividad en Colombiana no lo permite.

TAbla 3. Dosificaciones de Cantidades de Material Para $1 \mathrm{M}^{3}$ DE CONCRETo.

\begin{tabular}{|l|l|l|}
\hline \multicolumn{1}{|c|}{ Material } & Cantidad en $\mathrm{kg}$ & \multicolumn{1}{c|}{ Volumen en $\mathrm{m}^{3} / \mathrm{m}^{3}$} \\
\hline Cemento & 426 & 0,146 \\
\hline Agua & 195 & 0,205 \\
\hline Agregado grueso & 1001,07 & 0,394 \\
\hline Agregado fino & 614,95 & 0,235 \\
\hline Aire & 0,00 & 0,02 \\
\hline Total & 2237,02 & 1 \\
\hline
\end{tabular}

Fuente: Autores

Una vez se aplica la metodología descrita para el diseño de mezcla, se definieron las cantidades de materiales a emplear resumidas en la Tabla 3; de la cual se puede observar el empleo de una mezcla única, solo variando la cantidad de fibras [15]. De estas dosificaciones, se establece que el concreto fabricado es de una masa unitaria normal, no es de alto peso, poco poroso con una relación de $\mathrm{A} / \mathrm{mc}$ de 0,46 , para un concreto de alta plasticidad; asimismo se tiene una relación de cementos agregados del 26\%; la cual es alta, con la finalidad de obtener una buena adhesión entre cemento, agregados y fibras tal y como lo recomienda la ACI 544 3R-08 [16].

\section{B. Características mecánicas}

Una vez el concreto cumplió 56 días de maduración (se escogió esta fecha para garantizar que el cemento adquiera la resistencia más alta que puede brindar) se realizaron pruebas de resistencia a la flexión en concreto reforzado con fibras de acero [16] bajo la norma ASTMC-1609, resistencia a la compresión [17] bajo la norma ASTM C 39 y resistencia a la tensión indirecta [18] bajo la norma ASTM C 496 [19].

Es de aclarar, que para poder medir la velocidad de pulso ultrasónico en simultaneo a la deflexión de los elementos y la carga, fue necesario realizar modificaciones a los ensayos y equipos, fabricando elementos para la sujeción que permitiera sostener los palpadores del equipo de medición ultrasónico, diseñado por los autores del proyecto, esto permitió sujetar y medir en simultáneo con la carga, la deflexión y la velocidad de pulso ultrasónico propagada en el concreto en cada uno de las probetas fabricadas. Esto es observable en la Fig. 1 y Fig. 2. 


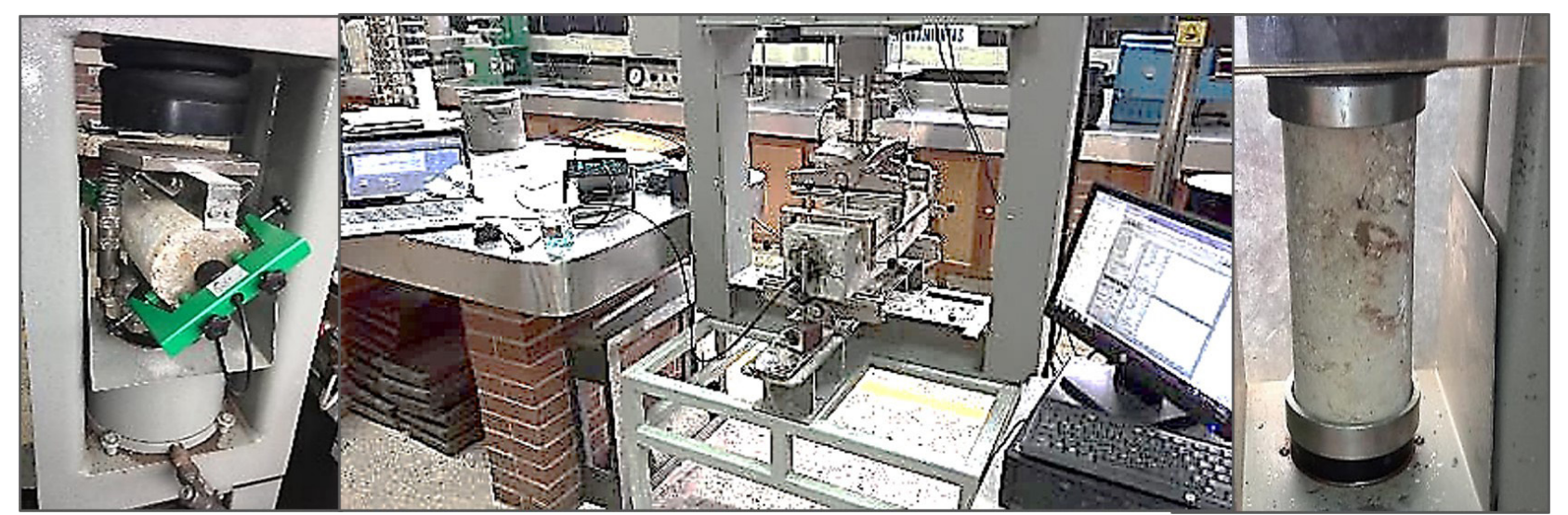

Fig. 1. Fotografías de los ensayos de resistencia a la tensión, flexión y compresión. Fuente: Autor.

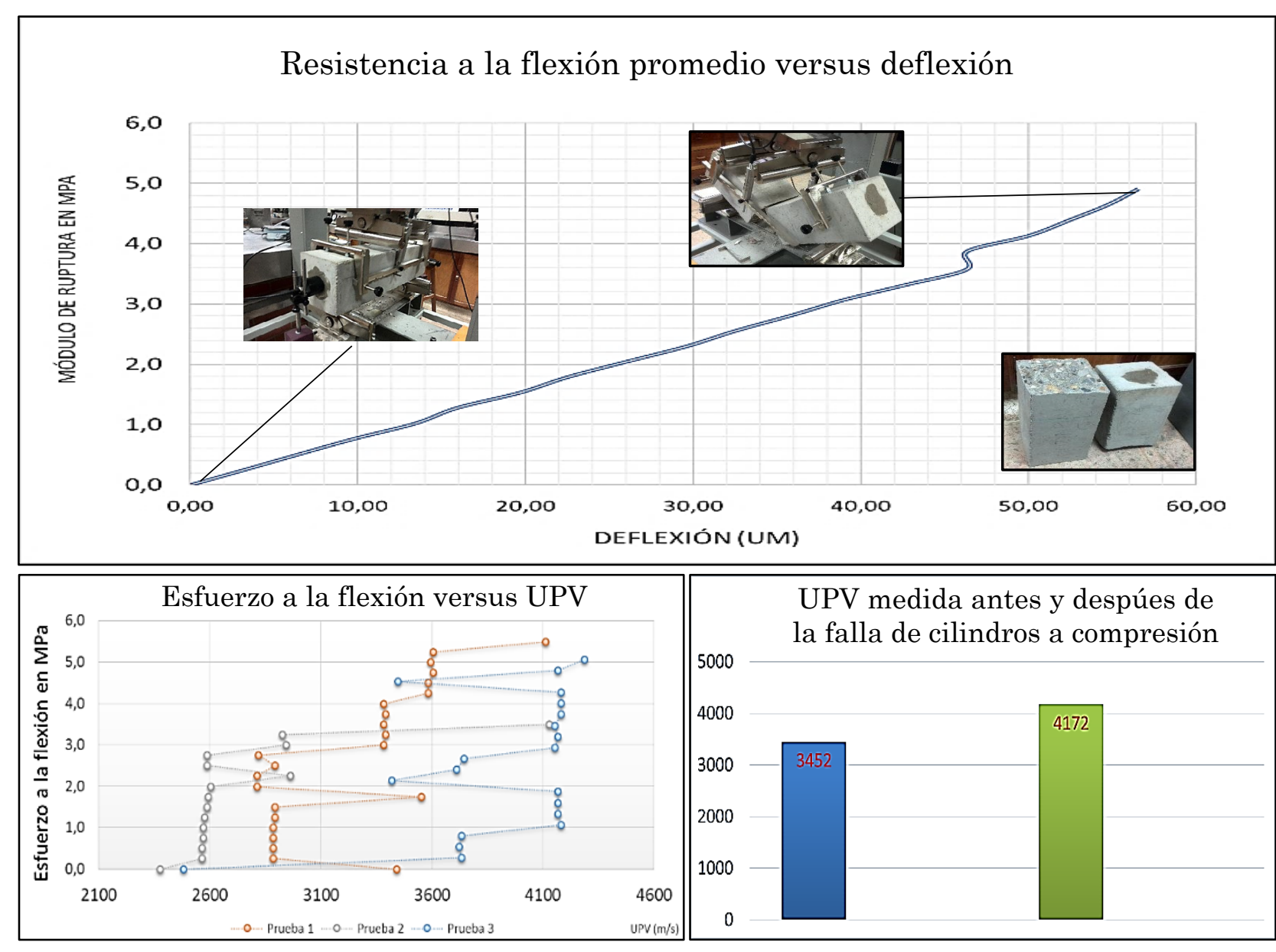

Fig. 2. Gráficas obtenidas en los ensayos ejecutados sobre las vigas con SFRC $0 \mathrm{~kg} / \mathrm{m}^{3}$. Fuente: Autor.

Asimismo, para realizar la medición de la deflexión, carga a la flexión y velocidad de pulso propagada, fue necesario emplear un sistema de adquisición de datos Controls MC 8 versión 5 y un software de medición de velocidad de pulso llamado Punditlink versión 2.4. Otro parámetro a tener en cuenta la velocidad del ensayo, con la finalidad de realizar mediciones en el mismo instante de tiempo, se calibró y ajusto el sistema de adquisición de datos con la velocidad de carga del equipo. El rango definido para la velocidad fue de $1 \mathrm{kN} / \mathrm{s}$, simultáneamente se realizó la medición en cada segundo la velocidad de pulso propagada logrando obtener estos parámetros en simultaneo.

\section{Resistencia a la Flexión}

\section{A. Mezcla SFRC $0 \mathrm{~kg} / \mathrm{m}^{3}$}

Las mezclas SFRC $0 \mathrm{~kg} / \mathrm{m}^{3}$, son aquellos que no contienen fibras de acero, en dicha mezcla se obtuvo una resistencia a la compresión de $57 \mathrm{MPa}$ [1] y un esfuerzo máximo a la flexión [2] promedio de 5,24 MPa, valores que ya son superiores al requisito de diseño. En cuanto a la velocidad de pulso ultrasónico se obtuvo una velocidad promedio de $3452 \mathrm{~m} / \mathrm{s}$; lo que expone que el concreto tiene una porosidad menor a la típica mezcla de concreto al obtener una velocidad superior a $3400 \mathrm{~m} / \mathrm{s}$ [8], lo que enfatiza en la buena calidad del concreto fabricado. En la Fig. 3 se exponen los resultados de este tipo de concreto. 


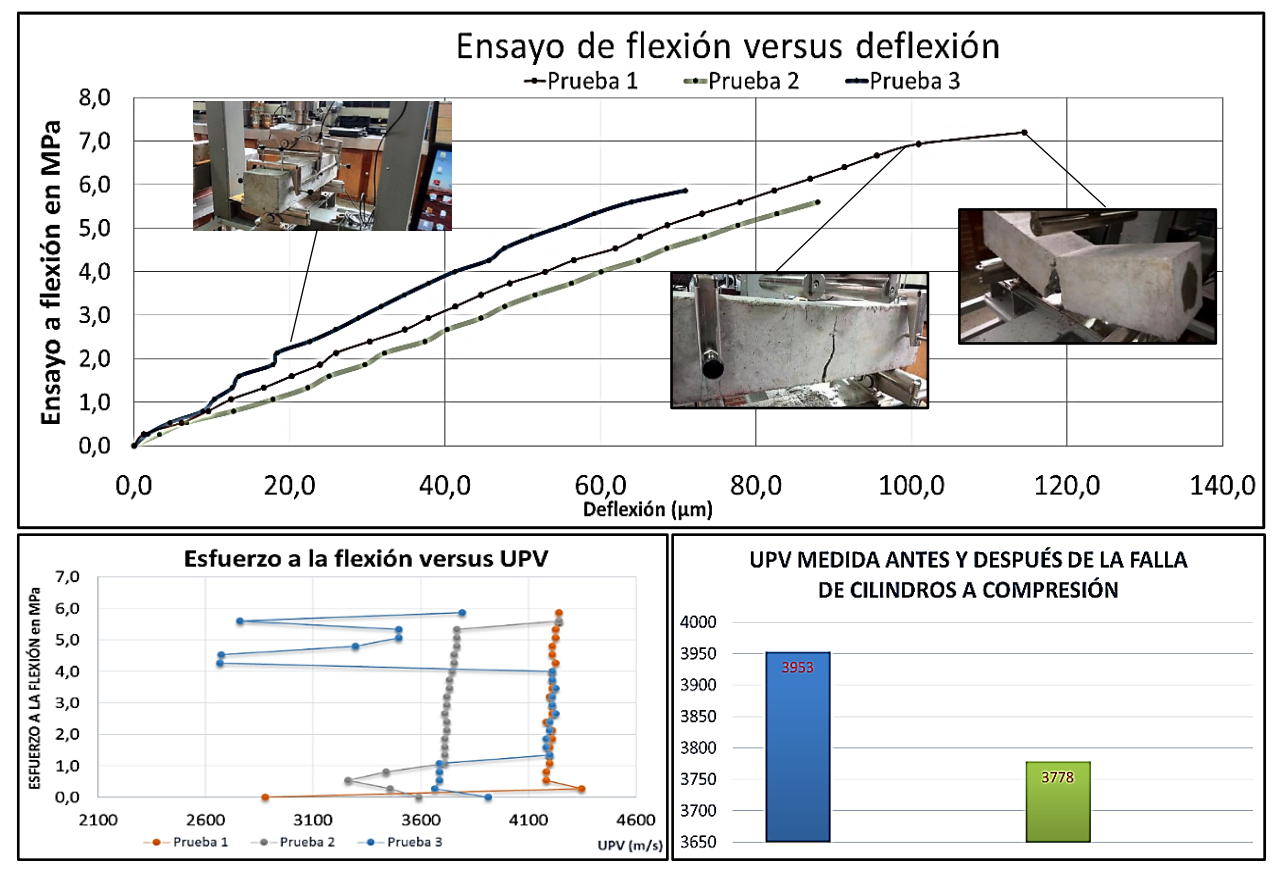

Fig. 3. Resultados obtenidos para el concreto SFRC $25 \mathrm{~kg} / \mathrm{m} 3$. Fuente: Autor.

\section{B. Mezcla SFRC $25 \mathrm{~kg} / \mathrm{m} 3$}

En las probetas realizadas con SFRC $25 \mathrm{~kg} / \mathrm{m}^{3}$, se encontró que el concreto aumenta su comportamiento mecánico siendo un material con mayor flexibilidad al concreto simple, por lo cual el material es más dúctil e inclusive es más fácil predecir el colapso del mismo, dado que no se genera falla explosiva; sin embargo, las fibras permitieron sostener la grieta generada por la falla y por lo tanto la viga no colapsa, ni rompe de manera frágil (comportamiento típico en el concreto simple).

La resistencia a la compresión disminuyó un $8 \%$ con respecto a la mezcla SFRC $0 \mathrm{~kg} / \mathrm{m}^{3}$ (53 MPa); en cuanto a la resistencia a la flexión está aumentó, obteniendo un valor de 7,20 MPa, misma situación se presentó para la resistencia a la tensión la cual fue de 4,85 MPa. Al observar los resultados, demuestra que las fibras trabajan a tensión y es por esto que se aumentan estas resistencias.

\section{Mezcla $S F R C 70 \mathrm{~kg} / \mathrm{m}^{3}$}

En la mezcla SFRC $70 \mathrm{~kg} / \mathrm{m}^{3}$, el concreto tuvo un comportamiento más elástico e inclusive luego de su fluencia se tuvo un comportamiento plástico, esto se puede observar en la Fig. 4. Igualmente, al momento de colapsar las vigas, estas se deformaban y no había un colapso inmediato; se notaron fisuras a lo largo del elemento, pero debido a la gran cantidad de fibras, la viga no colapsa y mantiene su integridad.

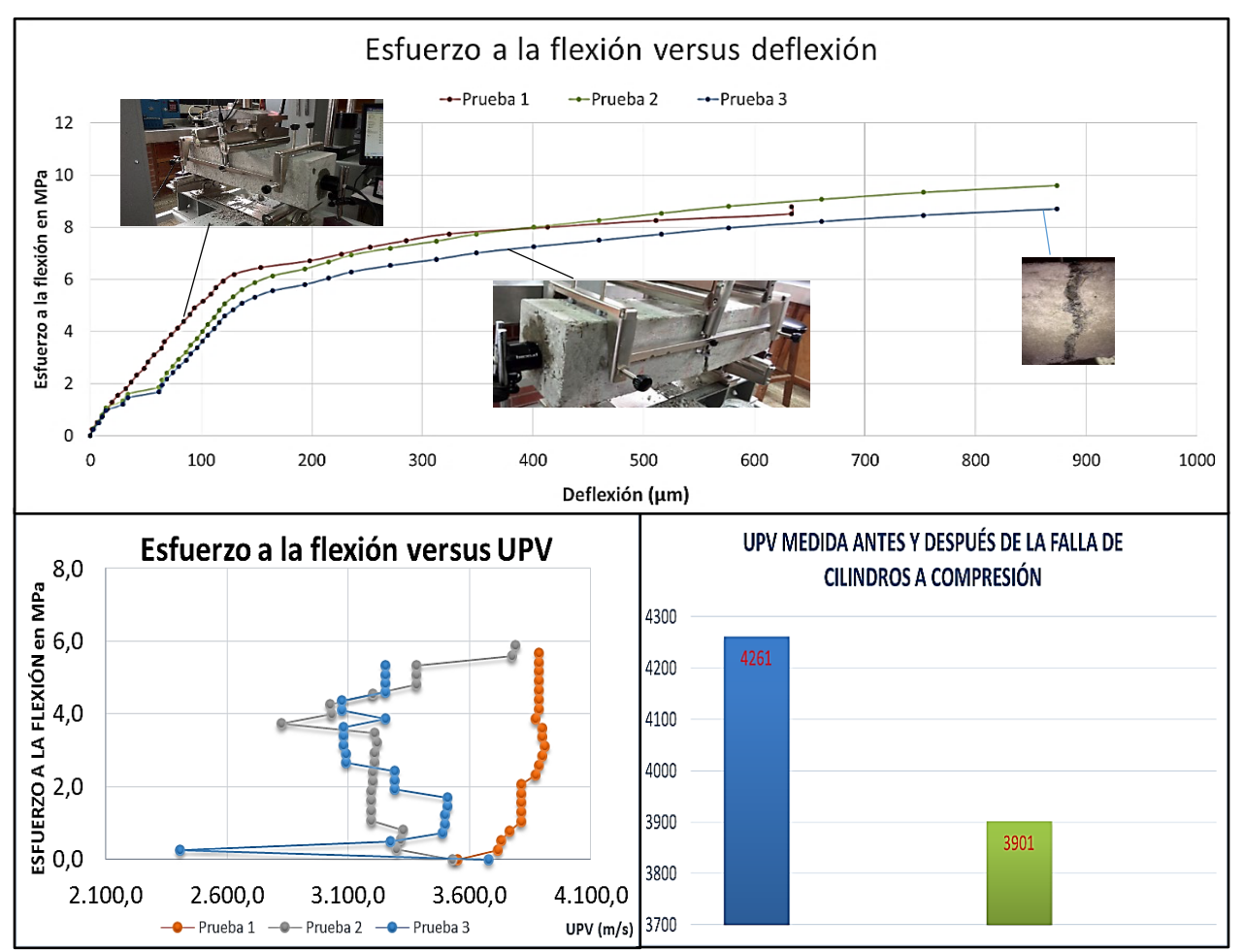

Fig. 4. Resultados obtenidos para el concreto SFRC 70 kg/m3. Fuente: Autor. 
Al observarla Fig. 4, es notable que el concreto tuvo una alta deflexión, ocasionando un comportamiento dúctil del mismo. Las resistencias máximas obtenidas en éste tipo de concretos fueron de $44 \mathrm{MPa}$ a la compresión, 8,42 MPa a la flexión y 5,80 MPa a tensión. Es claro que las fibras de acero están es para incrementar la resistencia a la flexión y tensión, pero al ocupar espacios de los áridos y reforzar la mezcla, ocasiona que la resistencia a la compresión se disminuya. Sin embargo, las ventajas que contrae el empelar alta cantidades de fibras es que el concreto tiene un comportamiento dúctil, el colapso del concreto es más predecible, además que aumenta la capacidad de presentar alto fisuramiento sin presentar colapso frágil, trayendo una mejor estabilidad de los elementos.

\section{Determinación del daño mecánico}

La velocidad de pulso ultrasónico depende principalmente de la densidad de los elementos construidos [8]; y a su vez, la densidad de un material depende de la cantidad de vacíos que contenga; es decir, si al medir la velocidad de onda propagada en el material es alta, la densidad también lo es; debido a que contiene menos espacios vacíos.

Basado en lo anterior se realizaron las mediciones de las velocidades de onda en las probetas elaboradas; obteniendo un resultado promedio de SFRC 0 kg/m³ de $2766 \mathrm{~m} / \mathrm{s}$; SFRC $25 \mathrm{~kg} / \mathrm{m}^{3}$ de $3459 \mathrm{~m} / \mathrm{s}$; SFRC $70 \mathrm{~kg} / \mathrm{m}^{3}$ de $3141 \mathrm{~m} / \mathrm{s}$. Al observar los resultados; la velocidad de onda más alta registrada en las mezclas fue para la segunda estableciendo que ésta fue la más densa. En cuanto a la que tenida mayor cantidad de fibras, la porosidad de los elementos aumentó y por lo tanto se disminuye la velocidad de onda propagada en el concreto.

Es de recordar que con la realización de los ensayos mecánicos se midió la velocidad de pulso ultrasónico, con los resultadovs obtenidos se realizó la Fig. 5. Al observar esta figura, en la mezcla SFRC $0 \mathrm{~kg} / \mathrm{m}^{3}$ el comportamiento fue lineal; mientras que en las otras mezclas hubo un cambio en su comportamiento, identificando dos tendencias; estas tendencias se dan por que el concreto con fibras adquiere un comportamiento dúctil, teniendo dos estados mecánicos, uno elástico y otro plástico, el cual se puede observar en las gráficas de esfuerzo versus deflexión en las mezclas SFRC $25 \mathrm{~kg} / \mathrm{m}^{3}$ y SFRC 70 kg/m³.

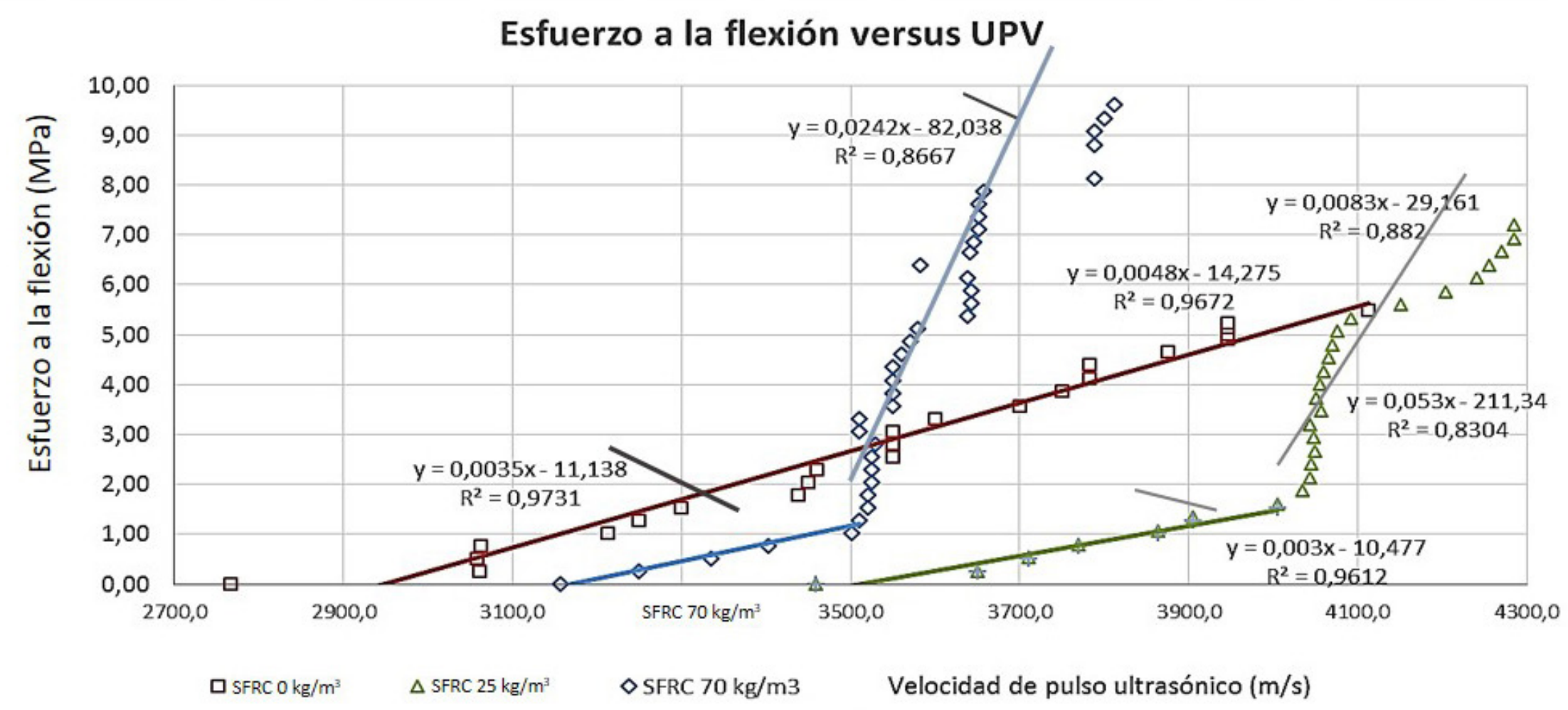

Fig. 5. Líneas de tendencia de los datos de Esfuerzo a la flexión versus UPV. Fuente: Autor.

Al analizar la Fig. 5, se realiza regresiones dependiendo del estado del material, y se plantea ecuaciones que servirán para predecir la resistencia a la flexión de éste tipo de concreto, de acuerdo a la velocidad de pulso ultrasónico mostrada. En la Tabla 4, se resume las ecuaciones obtenidas. 
TABla 4. Resumen de LAS ECUACIONES OBTEnidas.

\begin{tabular}{|l|l|}
\hline \multicolumn{1}{|c|}{ Mezcla Tipo } & \multicolumn{1}{|c|}{$\begin{array}{c}\text { Ecuaciones obtenidas } \\
\text { (el resultado da en MPa })\end{array}$} \\
\hline SFRC $0 \mathrm{~kg} / \mathrm{m}^{3}$ & $\begin{array}{l}\mathrm{y}=0,0048 \mathrm{x}-14,275 \\
\mathrm{R}^{2}=0,9672\end{array}$ \\
\hline SFRC $25 \mathrm{~kg} / \mathrm{m}^{3}$ rango elástico & $\begin{array}{l}\mathrm{y}=0,0035 \mathrm{x}-11,138 \\
\mathrm{R}^{2}=0,9731\end{array}$ \\
\hline SFRC $25 \mathrm{~kg} / \mathrm{m}^{3}$ rango plástico & $\begin{array}{l}\mathrm{y}=0,0242 \mathrm{x}-82,038 \\
\mathrm{R}^{2}=0,8667\end{array}$ \\
\hline SFRC $70 \mathrm{~kg} / \mathrm{m}^{3}$ rango elástico & $\begin{array}{l}\mathrm{y}=0,003 \mathrm{x}-10,477 \\
\mathrm{R}^{2}=0,9612\end{array}$ \\
\hline SFRC $70 \mathrm{~kg} / \mathrm{m}^{3}$ rango de fluencia & $\begin{array}{l}\mathrm{y}=0,053 \mathrm{x}-211,34 \\
\mathrm{R}^{2}=0,8304\end{array}$ \\
\hline SFRC $70 \mathrm{~kg} / \mathrm{m}^{3}$ rango plástico & $\begin{array}{l}\mathrm{y}=0,0083 \mathrm{x}-29,161 \\
\mathrm{R}^{2}=0,882\end{array}$ \\
\hline
\end{tabular}

Fuente: Autor.

\section{E. Evaluación del daño}

Para la evaluación del daño se empleó (2) y con los resultados de las mismas, se realizó la Fig. 6, en esta gráfica se puede observar que la tendencia del daño mecánico del concreto es totalmente lineal y que éste se puede observar que la tendencia del daño mecánico del concreto es totalmente lineal y que éste incrementa la velocidad y disminuye el daño con el uso de fibras de acero, debido al aumento de la resistencia a la flexión por la inclusión de éste material.

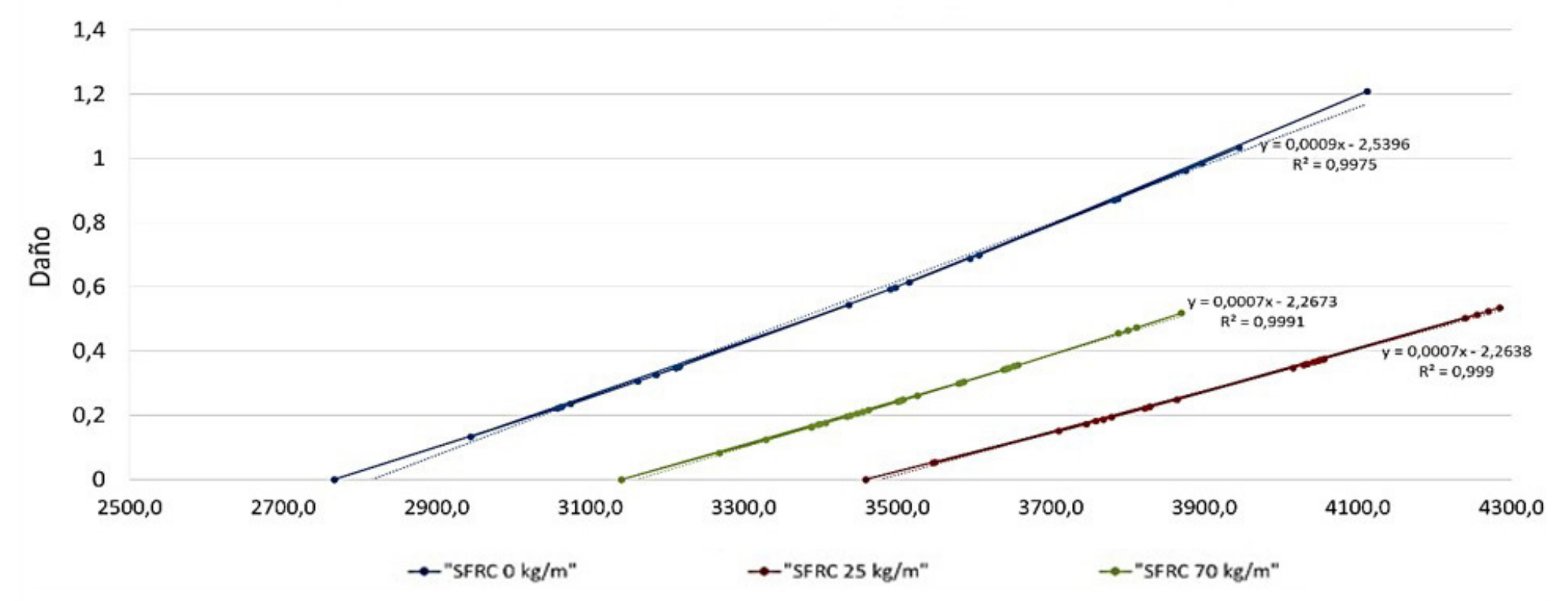

Fig. 6. Regresiones lineales a los datos obtenidos de Daño versus Velocidad de propagación. Fuente: Autor.

Sin embargo, cómo la mezcla que presentó mayor rigidez fue la de SFRC $25 \mathrm{~kg} / \mathrm{m}^{3}$, obtuvo un mejor comportamiento al daño del concreto y una de las mayores velocidades de onda medidas en el concreto. Igual que en las gráficas anteriores, se plantean ecuaciones que permiten medir el estado de deterioro mecánico y daño en elementos similares a los fabricados; esto se resumen en la Tabla 5. En estas ecuaciones se observa que el concreto al tener fibras, disminuye la pendiente de las mismas, lo que significa que el concreto mejorará su comportamiento dúctil a mayor cantidad de fibras.

Tabla 5. Ecuaciones obtenidas de la GRÁfica de daÑo versus velocidad de PUlso Ultrasónico.

\begin{tabular}{|l|l|}
\hline Mezcla Tipo & \multicolumn{1}{|c|}{$\begin{array}{c}\text { Ecuaciones obtenidas } \\
\text { (el resultado da en MPa) }\end{array}$} \\
\hline SFRC $0 \mathrm{~kg} / \mathrm{m}^{3}$ & $\begin{array}{l}\mathrm{y}=0,0009 \mathrm{x}-2,5396 \\
\mathrm{R}^{2}=0,9975\end{array}$ \\
\hline SFRC $25 \mathrm{~kg} / \mathrm{m}^{3}$ & $\begin{array}{l}\mathrm{y}=0,0007 \mathrm{x}-2,2673 \\
\mathrm{R}^{2}=0,9991\end{array}$ \\
\hline SFRC $70 \mathrm{~kg} / \mathrm{m}^{3}$ & $\begin{array}{l}\mathrm{y}=0,0007 \mathrm{x}-2,2638 \\
\mathrm{R}^{2}=0,999\end{array}$ \\
\hline
\end{tabular}

Fuente: Autor. 
Con estas ecuaciones dependiendo de la velocidad de pulso propagada en un elemento, puede predecirse en qué grado de daño mecánico se encuentra el concreto, para este tipo de mezclas. Para este caso y según las gráficas en un elemento sometido a flexión; al incrementarse la velocidad de onda propagada en elemento; este incrementará el daño del mismo; cuando el grado del daño sea igual a uno, el elemento ya no ofrece resistencia mecánica hacia la carga que éste soportando.

\section{Conclusiones}

De acuerdo con los resultados obtenidos y el análisis que se le ha dado a la información, a continuación, se establecen las conclusiones.

1. Las fibras de acero tienen un comportamiento dúctil, por lo cual al adherirse al concreto transmiten esta propiedad de manera proporcional, es decir entre mayor cantidad de fibras, se mejorará aún más la ductilidad del concreto, sin embargo, está ductilidad reduce la rigidez del mismo, trayendo como consecuencia una disminución de su resistencia a la compresión. Al momento de emplear las fibras, debe tenerse en cuenta esta característica.

2. De acuerdo al análisis de la información y los resultados de las pruebas mecánicas, las fibras de acero son un excelente refuerzo secundario del concreto, esto se demuestra al momento en el que se agrieta un elemento, las fibras de acero cosen la fisura presentada evitando que el elemento colapse de manera súbita y postergando su destrucción total; de esta manera aporta una resistencia post-fisuramiento que puede dar instantes de tiempo, antes del colapso.

3. De la misma manera, al tener un elemento más dúctil, el módulo elástico del elemento se reduce, igualmente para obtener una mezcla con esta propiedad se requiere mayor cantidad de fibras; en el caso de la mezcla SFRC $70 \mathrm{~kg} / \mathrm{m}^{3}$ fue la que obtuvo los elementos más dúctiles, esto se deduce de los diagramas de esfuerzo versus deflexión, en los cuales las gráficas de dicha mezcla están más inclinadas, demostrando lo anterior.

4. La principal utilidad de las fibras de acero es aumentar el módulo de ruptura del concreto, para lo cual en la mezcla SFRC $25 \mathrm{~kg} / \mathrm{m} 3$, aumentó la resistencia en un $24 \%$ con respecto a los elementos sin refuerzo, para las mezclas SFRC $70 \mathrm{~kg} / \mathrm{m}^{3}$ aumenta $43 \%$. Igualmente, estos elementos permiten tener un concreto dúctil, es decir concretos que permiten tener deflexiones de dos o tres veces más que el concreto sin refuerzo.

5. El comportamiento a tensión del concreto fue muy similar al comportamiento a flexión; en este caso las fibras de acero aumentaron la resistencia a la tensión, en especial en la mezcla de $70 \mathrm{~kg} / \mathrm{m} 3$, resultado esperado, debido a que las fibras de acero trabajan a tensión y por lo tanto es el estado de esfuerzo que más resiste las mismas. En la mezcla con mayor cantidad de fibras duplicó la resistencia con respecto al concreto simple.

6. Estos mismos efectos suceden con la tenacidad de los elementos; los que obtuvieron mejor capacidad de disipación de energía fueron los que tenían mezclas SFRC de $70 \mathrm{~kg} / \mathrm{m}^{3}$; teniendo unos valores de tenacidad 20 veces mayor a aquellos que no tenían refuerzo; haciendo de estos elementos más resistentes a cargas cíclicas y dinámicas.

7. Debe tenerse en cuenta que entre más fibras de acero contenga una mezcla de concreto, es más complicada de realizar, aumentando la precaución que se debe tener al momento de mezclado, inclusive con la mezcla de mayor cantidad de fibras, esta era muy complicada de moldear y fue necesario emplear más energía de moldeo y mezclado, para que los elementos quedarán bien fabricados.

8. En cuanto a la velocidad de pulso ultrasónico, en la medición de la misma en las probetas de concreto para los diferentes ensayos, esta se encontró entre $2500 \mathrm{~m} / \mathrm{s}$ hasta $3600 \mathrm{~m} / \mathrm{s}$, lo que indica que la densidad del concreto es normal. Igualmente se observada que al someterse los mismos a una carga, las velocidades de pulso ultrasónico fueron duplicadas aproximadamente; debido a que un elemento sometido a flexión aumenta su densidad al reducir los espacios vacíos del elemento y adicionalmente se reduce la distancia recorrida por la onda. Estos resultados son similares a lo obtenido en la investigación realizada por Bogas y Gómez. 
9. Asimismo, las fibras de acero influyen en la transmisión de la onda en un elemento; al hacer que elemento cambie su densidad y porosidad; por lo tanto, la transmisión de la velocidad de pulso ultrasónico varía considerablemente haciendo que las gráficas de esfuerzo vs. UPV tengan dos o tres cambios de pendiente, dependiendo. Lo que establece que la velocidad de pulso cambia de acuerdo al comportamiento mecánico de un material, es decir, si el mismo se encuentra en su estado elástico o plástico, conociéndose la condición en la que se encuentra un elemento. Esto se demuestra con las ecuaciones encontradas, las cuales dependiendo de la velocidad de onda registrada en el elemento; se puede establecer en qué grado de deterioro se encuentra; por ejemplo, cuando el valor se acerca a uno, el elemento se encuentra muy deteriorado.

10. Al determinarse el daño mecánico de los elementos; era de esperarse que las fibras de acero aumentará la resistencia de las mezclas; al punto que el elemento con mayor cantidad de fibras de acero fue el que más resistió, sin embargo las mezclas con SFRC $25 \mathrm{~kg} / \mathrm{m}^{3}$ tuvieron una resistencia frente al daño muy similar a los elementos de SFRC $70 \mathrm{~kg} / \mathrm{m}^{3}$, teniendo en cuenta que esta mezcla contiene menos de la mitad de fibras a la anterior; de esto se establece que la mezcla con SFRC $25 \mathrm{~kg} / \mathrm{m}^{3}$ es la dosificación más adecuada para reforzar el concreto, este planteamiento se afirma con la resistencia a la flexión y tensión obtenidas para estas mezclas.

11. Este efecto se puede observar en las ecuaciones obtenidas para la predicción del daño mecánico, las pendientes de los rangos elásticos y plásticos de las mezclas de $25 \mathrm{~kg} / \mathrm{m}^{3}$ y $70 \mathrm{~kg} / \mathrm{m}^{3}$, son muy parecidas (aproximadamente del 14\%) afirmando lo anterior. De aquí se recomienda que la mejor mezcla a emplear es la de $25 \mathrm{~kg} / \mathrm{m}^{3}$.

12. Las ecuaciones determinadas para evaluar el daño de una estructura son una primera afirmación y permitirán evaluar aproximadamente el daño de una estructura de concreto; de acuerdo a Lemaitre una medición del grado de daño menor a 0,5; es un elemento que probablemente no va a colapsar, sin embargo, si la medición del grado del daño es superior a 0,5 se encuentra con una probabilidad de que la estructura se encuentre con fisuras que impedirán que la misma soporte un estado de esfuerzos mayor al que está sometido y por lo tanto puede colapsar.

\section{Agradecimientos}

Los autores de ésta investigación agradecen el apoyo en conocimientos y financieros a la Universidad Militar Nueva Granada y a la Universidad Nacional de Colombia de la cual se derivó este artículo.

\section{REFERENCIAS}

[1] ASTM, Standard Test Method for Compressive Strength of Cylindrical Concrete Specimens. West Conshohocken, USA: ASTM, American Society for Testing and Materials, 2017.

[2] ACI, Standard Practice for Selecting Proportions for Normal, Heavyweight and Mass Concrete ACI 211.1. Chicago, USA: ACI, American Concrete Institute, 2009.

[3] J. Lemaitre, Mechanics of Solid Materials. Cambridge, USA: CU, 2009.

[4] D. Sánchez, Tecnología del Concreto. Bogotá D.C., CO: Bhandar Editores, 2001.

[5] P. Marmol, "Hormigones con fibras de acero. caracteristicas mecanicas", M. S. Thesis. Depto. Ing. Civ. UPM, Madrid, ES, 2010.

[6] M. Á. Ospina, L. Á. Moreno \& K. A. Rodríguez, "Technical-economic analysis about use the recycle concrete, comparative with convetional concrete in Colombia", Actas Ing., vol. 3, pp. 36-47, Aug. 2017.

[7] G. Jianming, W. Sun \& K. Morino, "Mechanical Propierties of Steel Fiber-reinforced, High-strength, Lightweight Concrete", Cem. Concr. Compos., vol. 19, no. 4, pp. 307-313, Aug. 2007. https://doi.org/10.3390/ ma12152470

[8] J. A. Bogas, M. G. Gómez \& A. Gómez, "Compressive strength evaluation of structural light weight Concrete by non-destructive ultrasonic pulse velocity method", ScienceDirect, vol. 53, no. 5, pp. 962-972, Jul. 2013. https://doi.org/10.1016/j.ultras.2012.12.012

[9] J. León, J. Lizarazo \& J. Carrillo, "Material Damage Evolution for Plain and Steel-Fiber-Reinforced", AJSE, vol. 43, pp. 1-9, May. 2018.

[10] R. Carcaño, E. Moreno \& W. Castillo, "Predicción de la resistencia del concreto con base en la velocidad de pulso ultrasónico y un índice de calidad de los agregados", Ing. Rev. Académic., vol. 8, no. 2, pp. 41-52, Jan. 2004. 
[11] X. Guan, X. Liu, X. Jia, Y. Yuan, J. Cui \& H. Mang, "A stochastic multiscale model for predicting mechanical propierties of fiber reinforced concrete", Intl. J. Solids. Struct., vol. 56-57, pp. 280-289, Mar. 2015. https://doi.org/10.1016/j.jijsolstr.2014.10.008

[12] P. Song \& S. Hwang, "Mechanical propierties of high.strengh steel fiber-reinforced Concrete", Constr. Build. Mater, vol. 18, no. 9, pp. 669-673, Nov. 2004. https://doi.org/10.1016/j.conbuildmat.2004.04.027

[13] ARGOS, Tecnología del Concreto y sus Componentes. Bogotá D. C.: Asocreto, 2010.

[14] Y. Ding \& K. Wolfgang, "Comparative study of steel fibre-reinforced concrete and steel mesh-reinforced concrete at early ages in panel tests", Cem. Concr. Res, vol. 29, no. 11, pp. 1827-1834, Nov. 1999. https:// doi.org/10.1016/S0008-8846(99)00177-5

[15] J. Karadelis \& Y. Lin, "Flexural strengths and fibre efficiency of steel-fibre-reinforced, roller-compacted, polymer modified concrete", Constr. Build. Mater, vol. 93, pp. 498-505, Sept. 2015.

[16] ACI, Guide for Specifying, Proportioning, and Production of Fiber-Reinforced Concrete. ACI 544.4 R-08. Farmington Hills, USA: ACI, American Concrete Institute, 2008

[17] ASTM, Standard Test Method for Flexural Performance of Fiber-Reinforced Concrete (Using Beam With Third-Point Loading). ASTM-C-1609. Philadelphia, USA: ASTM, American Society for Testing and Materials, 2012.

[18] ASTM, Standard Test Method for Compressive Strength of Cylindric Concrete Specimens. ASTM C39. Philadelphia, USA: ASTM, American Society for Testing and Materials, 2012.

[19] ASTM, Standard Test Method for Determination of Diametrical Compressive Tensile Strength of Cylindrical Concrete Specimens. ASTM C496/C496M-17. Philadelphia, USA: ASTM, American Society for Testing and Materials, 2012.

[20] AIS, Reglamento Colombiano de construcción sismo resistente. NSR-10. Bogotá, D. C.: AIS, Asociación Colombiana de Ingeniería Sísmica, 2010.

Miguel Ángel Ospina García es Ingeniero Civil, especialista en Gerencia de la Calidad, Magister en Construcción, docente de tiempo completo del programa de Ingeniería Civil a distancia de la Universidad Militar Nueva Granada (Cajicá, Colombia). Sus principales áreas de investigación y desempeño, es en el estudio de materiales en la construcción y en metodologías para el aseguramiento y gestión de la calidad constructivas. https://orcid.org/0000-0003-3700-7495

Juan Manuel Lizarazo Marriaga es Ingeniero Civil, especialista en estructuras, Magister en Estructuras, Magister en Ciencias de la Construcción, Doctor en Ingeniería Civil, docente de tiempo completo del programa de Ingeniería Civil de la Facultad de Ingeniería y agronomía de la Universidad Nacional de Colombia (Bogotá, D.C., Colombia). Sus áreas de investigación y desempeño son en la ingeniería de estructuras de edificaciones y el empleo de materiales en la construcción. https://orcid.org/0000-0002-6543-1640

Andrés Salas Montoya es Ingeniero Civil, Doctor en Ingeniería, Post Doctor en Ingeniería Civil, docente de tiempo completo del programa de Ingeniería Civil (Manizales, Colombia). Sus áreas de investigación y desempeño son en el estudio sobre la durabilidad y sostenibilidad de materiales constructivos. https://orcid.org/0000-0003-2779-1634 